\title{
Firefly Algorithm Approach For Rational Bézier Border Reconstruction of Skin Lesions from Macroscopic Medical Images
}

\author{
Akemi Gálvez \\ Dept. of Information Science \\ Faculty of Sciences \\ Toho University \\ Funabashi, Japan \\ Dept. Appl. Math. \& Comp. Sci. \\ University of Cantabria \\ Santander, Spain \\ galveza@unican.es \\ Lihua You \\ National Center for Computer Animation \\ Bournemouth University \\ The Media School, Talbot Campus \\ Poole, UK \\ LYou@bournemouth.ac.uk
}

\author{
Andrés Iglesias \\ Dept. of Information Science \\ Faculty of Sciences \\ Toho University \\ Funabashi, Japan \\ Dept. Appl. Math. \& Comp. Sci. \\ University of Cantabria \\ Santander, Spain \\ iglesias@unican.es
}

\author{
Habibollah Haron \\ Dept. of Computer Science \\ Faculty of Computing \\ Universiti Teknologi Malaysia \\ Skudai, Malaysia \\ habib@utm.my
}

\author{
Hassan Ugail \\ Faculty of Engineering \& Informatics \\ Media Design and Technology \\ University of Bradford \\ Bradford, UK \\ H.Ugail@bradford.ac.uk
}

\begin{abstract}
Image segmentation is a fundamental step for image processing of medical images. One of the most important tasks in this step is border reconstruction, which consists of constructing a border curve separating the organ or tissue of interest from the image background. This problem can be formulated as an optimization problem, where the border curve is computed through data fitting procedures from a collection of data points assumed to lie on the boundary of the object under analysis. However, standard mathematical optimization techniques do not provide satisfactory solutions to this problem. Some recent papers have applied evolutionary computation techniques to tackle this issue. Such works are only focused on the polynomial case, ignoring the more powerful (but also more difficult) case of rational curves. In this paper, we address this problem with rational Bézier curves by applying the firefly algorithm, a popular bio-inspired swarm intelligence technique for optimization. Experimental results on medical images of melanomas show that this method performs well and can be successfully applied to this problem.

Index Terms - medical images, image segmentation, border reconstruction, skin lesions, swarm intelligence, firefly algorithm, rational curves, Bézier curves
\end{abstract}

\section{INTRODUCTION}

\section{A. Motivation}

Computer-aided detection and reconstruction of the boundaries of objects and areas in images has been a hot topic of research for decades. It is a recurrent problem in fields such as image processing, pattern recognition, artificial vision,

Work funded by EU Horizon 2020 research and innovation programme (PDE-GIR project, MSCA grant agreement No 778035) and grant \#TIN201789275-R of Spanish Agency of Research (AEI) and European Funds EFRD. and virtual and augmented reality, among many others. It is also a popular and increasingly used technology for medical applications. For instance, it is widely applied to identify and discriminate different tissues and organs for medical visualization in popular non-invasive diagnostic procedures such as computer tomography, magnetic resonance imaging, infrared imaging, positron emission tomography, ultrasonography, spectroscopy, and so on.

One of its most interesting applications in medicine arises in dermoscopy, for early detection and efficient treatment of melanoma and other skin lesions [19], [23]. This is critical issue in current medical and healtcare systems all over the world. According to some reliable studies of the International Agency for Research Cancer of the World Health Organization, one in 5 men and one in 6 women worldwide will develop cancer during their lifetime, and one in 8 men and one in 11 women will die from the disease. It has also been reported that more than 90 million people has been affected by cancer in 2015, with more than 15 million cases arising every year. These figures are still raising, with 18.1 million new cases and 9.6 million deaths in 2018.

Skin cancer is one of the most frequent types of cancer for both men and women. According to the World Cancer Report 2014, melanoma is the most frequent and dangerous type of skin cancer. In this case, the classical reasons for cancer (population growth, ageing, bad habits, etc.) are strengthened by new social customs. For instance, the popularity of sunlight exposure is linked to a dramatic increase in the number of 
cases of skin cancer and other skin diseases. According to a report from the Australian Cancer Council: "Almost all skin cancers (approximately 99\% of non-melanoma skin cancers and $95 \%$ of melanoma) are caused by too much $U V$ radiation from the sun or other sources such as solaria (solariums, sunbeds, and sun lamps)."

As a result, healthcare systems are stringently asked for diligent and accurate diagnosis and treatment of skin lesions. Time is a key factor in this process, as early detection is critical for an efficient treatment of melanoma and other malignant skin lesions. It has been widely reported that the five-year survival rate is about $99 \%$ for stage 0 melanoma (in situ), when the tumor is confined to the epidermis, while it is only $7 \% \sim 20 \%$ for stage 4 melanoma, when the cancer has spread to other parts of the body.

Visual inspection by a specialist is the most common diagnostic procedure. However, it is difficult to distinguish the melanoma from other skin lesions. Other diagnosis procedures include the ABCDE method, the Menzies scale, the 7-point checklist, and different types of biopsy [8], [22]. These procedures rely heavily on human intervention, leading to diagnostic results that can vary significantly even among very experienced dermatologists. Image-based methods are gaining popularity in the field in recent years. The most classical one is dermoscopy. where images are digitally captured in an process called digital epiluminescence dermoscopy. Dermoscopy is more precise than naked eye examination in about $20 \%$ for detection of melanomas and about $10 \%$ for percentage of non-melanomas correctly diagnosed as benign [3]. Unfortunately, it is also prone to errors due to the difficulty and subjectivity of visual interpretation of images.

The standard approach in automatic medical image processing of skin lesions consists of three stages: 1) image segmentation; 2) feature extraction and feature selection; and 3) lesion classification. Image segmentation aims to identify the area of the skin lesion and separate it from the background. This stage is very important, as it represents the starting point for the whole pipeline and it affects the accuracy of the next stages. Popular segmentation approaches include thresholding methods [4], [16], edge-based methods [2], clustering methods [25], [29], level set methods [21] and active contours [20], among others.

An important task in segmentation is the border detection of the skin lesion from the image. This is a valuable source of information for accurate diagnosis, as several clinical features can be computed directly from the detected border (for instance, irregular borders are a good indicator of possible malignant tumors). Until recently, the border detection was handled manually by dermatologists by clicking with the mouse on different parts of the image on a computer screen to obtain an initial collection of feature points joined with linear segments. However, the resulting polyline is not well suited for this process, as the border of skin lesions rarely happens to be piecewise linear, but smooth. Given the input data, parametric approximation schemes are clearly better suited for this task. This is the main motivation of the present work.
A previous paper by some of the authors presented a swarm intelligence approach for dealing with this problem using Bézier curves [15]. The method works well and provides satisfactory results in many cases. However, it was pointed out that the accuracy and applicability of the process can be improved by using more powerful and sophisticated basis functions. In this work, we follow this approach by replacing the polynomial basis functions by rational ones. In this case, we have extra parameters called weights, which allows the user to modify the shape of the curve locally by simply changing the weight of one or several poles of the curve without moving the location of the poles at all. This is an interesting and valuable feature, as it makes it possible to reduce the degree of the curve significantly without penalizing the approximation accuracy. The resulting parametric curve is no longer a polynomial but a rational function.

Unfortunately, using rational curves is by far much more difficult than the polynomial case, because some extra variables (the weights) have also to be computed. In addition, the different variables (data parameters, poles, and weights) are strongly related to each other in a highly nonlinear way [5]. As a result, we have to solve a difficult continuous multivariate nonlinear optimization problem that cannot be properly solved in the general case through traditional mathematical optimization techniques. Our method to solve this problem is based on a nature-inspired metaheuristics called firefly algorithm, introduced by Prof. X.S. Yang to solve difficult optimization problems [26]. To the best of our knowledge, this method has never been used so far for border reconstruction of medical images with rational curves.

The structure of this paper is as follows: the problem to be solved is discussed in detail in Section III. The fundamentals and main features of the firefly algorithm are discussed in Section III. The proposed method to solve the border reconstruction problem is presented in Section IV. To illustrate the performance of our method, it is applied in Section V to perform border reconstruction of two medical images. The paper closes in Section VI with the main conclusions of this contribution and our plans for future work in the field.

\section{THE PROBLEM}

The problem to be solved in this paper can be formulated as follows: suppose that we are provided with a sorted collection of feature points $\left\{\boldsymbol{\Delta}_{i}\right\}_{i=1, \ldots, \kappa}$ in $\mathbb{R}^{2}$ obtained from medical images by a trained dermatologist. Such feature points correspond to the boundary curve between a skin lesion or tumor and the skin background. Note that in this paper vectors are denoted in bold. Since the feature points are collected in a manual way, they are subjected to measurement noise, irregular sampling, and other artifacts. Therefore, an approximation scheme based on a smooth mathematical curve is generally more suitable for border detection than the linear interpolation given by a polyline connecting the feature points through simple straight lines. Consequently, our goal is to compute a parametric curve $\boldsymbol{\Phi}(\tau)$ performing discrete approximation of the feature points $\left\{\boldsymbol{\Delta}_{i}\right\}_{i}$ in the least-squares sense. 
Several families of approximation functions can be used for this task. Among them, the free-form parametric curves (such as Bézier and B-spline curves) are popular choices, because of their flexibility and wide applicability in academia and industrial settings [5], [24]. Some previous papers have addressed the case of Bézier curves using different swarm intelligence methods, such as the bat algorithm [14] and the cuckoo search algorithm [15]. However, in all these cases, only the polynomial case has been considered, while surprisingly the (more powerful) rational case has been ignored so far. This paper aims at filling this gap. In particular, in this work we focus on the case of rational Bézier curves [7], [24].

Mathematically, a free-form rational Bézier curve $\mathbf{\Phi}(\tau)$ of degree $\eta$ is defined as:

$$
\boldsymbol{\Phi}(\tau)=\frac{\sum_{j=0}^{\eta} \omega_{j} \boldsymbol{\Lambda}_{j} \phi_{j}^{\eta}(\tau)}{\sum_{j=0}^{\eta} \omega_{j} \phi_{j}^{\eta}(\tau)}
$$

where $\boldsymbol{\Lambda}_{j}$ are vector coefficients called the poles, $\omega_{j}$ are their scalar weights, $\phi_{j}^{\eta}(\tau)$ are the Bernstein polynomials of index $j$ and degree $\eta$, given by:

$$
\phi_{j}^{\eta}(\tau)=\left(\begin{array}{c}
\eta \\
j
\end{array}\right) \tau^{j}(1-\tau)^{\eta-j}
$$

with $\left(\begin{array}{l}\eta \\ j\end{array}\right)=\frac{\eta !}{j !(\eta-j) !}$, and $\tau$ is the curve parameter, defined on the finite interval $[0,1]$. By convention, $0 !=1$.

Now, our optimization problem consists of computing all parameters (i.e. poles $\boldsymbol{\Lambda}_{j}$, weights $\omega_{j}$, and parameters $\tau_{i}$ associated with the $\boldsymbol{\Delta}_{i}$, for $\left.i=1, \ldots, \kappa, j=0, \ldots, \eta\right)$ of the rational Bézier curve $\boldsymbol{\Phi}(\tau)$ approximating the feature points better in the least-squares sense. This means minimizing the least-squares error, $\Upsilon$, defined as the sum of squares of the residuals:

$$
\Upsilon=\underset{\substack{\left\{\tau_{i}\right\}_{i} \\\left\{\Lambda_{j}\right\}_{j} \\\left\{\omega_{j}\right\}_{j}}}{\operatorname{minimize}}\left[\sum_{i=1}^{\kappa}\left(\boldsymbol{\Delta}_{i}-\frac{\sum_{j=0}^{\eta} \omega_{j} \boldsymbol{\Lambda}_{j} \phi_{j}^{\eta}\left(\tau_{i}\right)}{\sum_{j=0}^{\eta} \omega_{j} \phi_{j}^{\eta}\left(\tau_{i}\right)}\right)^{2}\right] .
$$

Now, taking:

$$
\varphi_{j}^{\eta}(\tau)=\frac{\omega_{j} \phi_{j}^{\eta}(\tau)}{\sum_{k=0}^{\eta} \omega_{k} \phi_{k}^{\eta}(\tau)}
$$

Eq. (2) becomes:

$$
\Upsilon=\underset{\substack{\left\{\tau_{i}\right\}_{i} \\\left\{\Lambda_{j}\right\}_{j} \\\left\{\omega_{j}\right\}_{j}}}{\operatorname{minimize}}\left[\sum_{i=1}^{\kappa}\left(\boldsymbol{\Delta}_{i}-\sum_{j=0}^{\eta} \boldsymbol{\Lambda}_{j} \varphi_{j}^{\eta}(\tau)\right)^{2}\right],
$$

which can be rewritten in matrix form as:

$$
\boldsymbol{\Omega} . \boldsymbol{\Lambda}=\boldsymbol{\Xi}
$$

called the normal equation, where:

$$
\begin{gathered}
\boldsymbol{\Omega}=\left[\Omega_{i, j}\right]=\left[\left(\sum_{k=1}^{\kappa} \varphi_{i}^{\eta}\left(\tau_{k}\right) \varphi_{j}^{\eta}\left(\tau_{k}\right)\right)_{i, j}\right], \\
\boldsymbol{\Xi}=\left[\Xi_{j}\right]=\left[\left(\sum_{k=1}^{\kappa} \boldsymbol{\Delta}_{k} \varphi_{j}^{\eta}\left(\tau_{k}\right)\right)_{j}\right],
\end{gathered}
$$

$\boldsymbol{\Lambda}=\left(\boldsymbol{\Lambda}_{0}, \ldots, \boldsymbol{\Lambda}_{\eta}\right)^{T}$, for $i, j=0, \ldots, \eta$, and $(.)^{T}$ means the transposition of a vector or a matrix. In general, $\kappa>>\eta$ meaning that the system (5) is over-determined. If values are assigned to the $\tau_{i}$, our problem can be solved as a classical linear least-squares minimization, with the coefficients $\left\{\boldsymbol{\Lambda}_{i}\right\}_{i=0, \ldots, \eta}$ as unknowns. This problem can readily be solved by standard numerical techniques. On the contrary, if the values of $\tau_{i}$ are treated as unknowns, the problem becomes much more difficult. Indeed, since the polynomial blending functions $\phi_{j}^{\eta}(\tau)$ are nonlinear in $\tau$ and so are the rational blending functions $\varphi_{j}^{\eta}(\tau)$, the least-squares minimization of the errors is a nonlinear continuous optimization problem. Note also that in many practical cases the number of data points can be extremely large, meaning that we have to deal with a large number of unknowns. In other words, we are dealing with a high-dimensional problem. It is also a multimodal problem, since there might be more than one set of parameter values leading to the optimal solution.

To summarize, the interplay among all sets of unknowns (data parameters, poles, and weights) leads to a very difficult over-determined, multimodal, multivariate, continuous, nonlinear optimization problem. In this work, we are interested to solve this general problem. Therefore, instead of making assumptions about the values of the free parameters, we are aimed at computing all of them in an integrated way.

Unfortunately, it has been proved that the classical mathematical optimization methods are not able to solve this difficult problem. As a consequence, there has been a great interest to explore other alternatives, including artificial intelligence techniques. Among them, the evolutionary computation methods of biological inspiration are very powerful tools for continuous optimization [1], [6], [28], including medical applications [18]. For instance, as discussed above, swarm intelligence methods have already been applied to quite close (but simpler) problems, such as the border detection problem with polynomial curves [12], [14], [15] and more complicated problems [9][11], [13], [17]. Encouraged by these positive results, we follow a similar strategy here for this (more difficult) rational problem. In particular, we address our optimization problem by using a powerful bio-inspired method called firefly algorithm and described in next section.

\section{The Firefly Algorithm}

The firefly algorithm (FFA) is a nature-inspired metaheuristic algorithm introduced in 2008 to solve optimization problems [26], [27]. The algorithm is based on the social flashing behavior of fireflies in nature. The key ingredients of the 
method are the variation of light intensity and formulation of attractiveness. In general, the attractiveness of an individual is assumed to be proportional to their brightness, associated with the objective function of the given optimization problem.

\section{A. Basic rules}

In the firefly algorithm, there are three particular idealized rules, which are based on some of the major flashing characteristics of real fireflies [26]. They are:

1) all fireflies are unisex, so that one firefly will be attracted to other fireflies regardless of their sex;

2) The degree of attractiveness of a firefly is proportional to its brightness, which decreases as the distance from the other firefly increases due to the fact that the air absorbs light. For any two flashing fireflies, the less brighter one will move towards the brighter one. If there is not a brighter or more attractive firefly than a particular one, it will then move randomly;

3) The brightness or light intensity of a firefly is determined by the value of the objective function of a given problem. For instance, for maximization problems, the light intensity can simply be proportional to the value of the objective function.

The distance between any two fireflies $i$ and $j$, at positions $\mathbf{s}_{i}$ and $\mathbf{s}_{j}$ at time instance $t$, respectively, can be defined as a Cartesian or Euclidean distance as follows:

$$
r_{i j}^{t}=\left\|\mathbf{s}_{i}^{t}-\mathbf{s}_{j}^{t}\right\|=\sqrt{\sum_{k=1}^{d}\left(s_{i, k}^{t}-s_{j, k}^{t}\right)^{2}}
$$

where $s_{i, k}^{t}$ is the $k$-th component of the spatial coordinate $\mathbf{s}_{i}^{t}$ of the $i$-th firefly and $d$ is the number of dimensions.

In the firefly algorithm, as attractiveness function of a firefly $j$ one should select any monotonically decreasing function of the distance to the chosen firefly, e.g., the exponential function:

$$
\beta=\beta_{0} e^{-\gamma\left(r_{i j}^{t}\right)^{2}}
$$

where $r_{i j}^{t}$ is the distance defined as in Eq. (6), $\beta_{0}$ is the initial attractiveness at $t=0$, and $\gamma$ is an absorption coefficient at the source that controls the decrease of the light intensity.

The movement of a firefly $i$ at time $t+1$ which is attracted by a more attractive (i.e., brighter) firefly $j$ is governed by the following evolution equation:

$$
\mathbf{s}_{i}^{t+1}=\mathbf{s}_{i}^{t}+\beta_{0} e^{-\gamma\left(r_{i j}^{t}\right)^{2}}\left(\mathbf{s}_{j}^{t}-\mathbf{s}_{i}^{t}\right)+\alpha\left(\sigma-\frac{1}{2}\right)
$$

where the first term on the right-hand side is the current position of the firefly, the second term is used for considering the attractiveness of the firefly to light intensity seen by adjacent fireflies, and the third term is used for the random movement of a firefly in case there are not any brighter ones. The coefficient $\alpha$ is a randomization parameter determined by the problem of interest, while $\sigma$ is a random number uniformly distributed on the interval $[0,1]$.

From Eq. (8), the firefly algorithm is driven by three parameters: the randomization parameter $\alpha$, the attractiveness

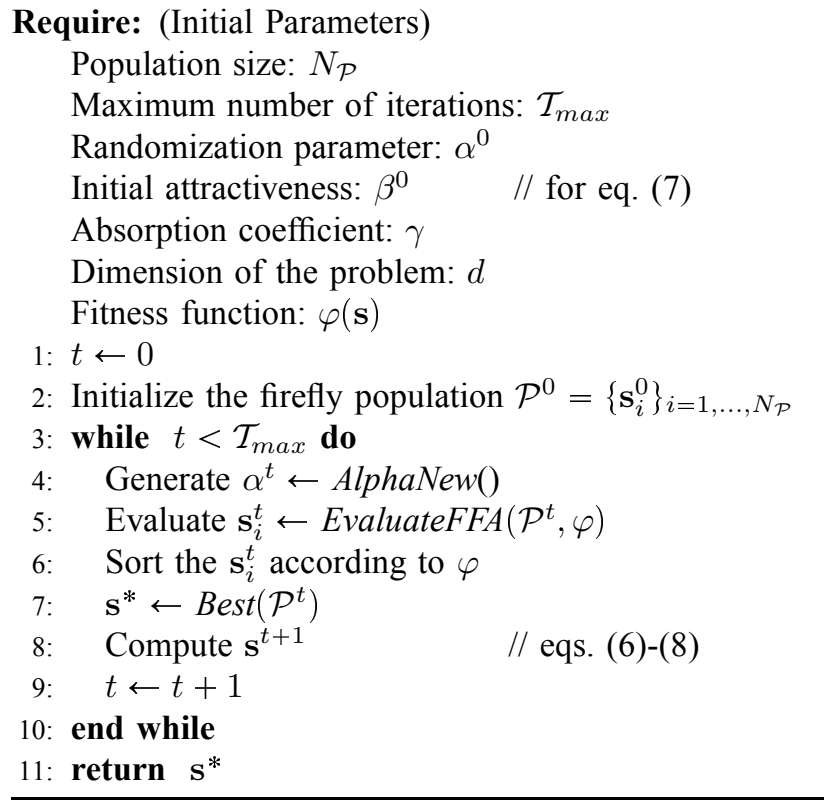

Algorithm 1: Firefly algorithm pseudocode

$\beta$ and the absorption coefficient $\gamma$. Depending on their values, the algorithm exhibits two asymptotic behaviors, for $\gamma \rightarrow 0$ and $\gamma \rightarrow \infty$. In the former case $\gamma \rightarrow 0$, we have $\beta=\beta_{0}$ according to Eq. (7), meaning that the attractiveness is constant throughout the search space. This behavior is a special case of the particle swarm optimization (PSO) method, in particular, the accelerated particle swarm optimization if we replace $\mathbf{s}_{j}$ by $\mathbf{s}^{*}$. In the later case $\gamma \rightarrow \infty$, the second term in Eq. (8) vanishes and the algorithm becomes essentially a random walk, kind of a parallel version of simulated annealing (SA). This behavior also happens for $\beta_{0}=0$ regardless of the value of $\gamma$. Furthermore, if we set $\gamma=0$ and $\beta_{0}=1$, the algorithm becomes a simplified version of differential evolution (DE) without mutation with the crossover rate controlled by $\beta_{0}$. This means that the firefly algorithm includes PSO, SA, and $\mathrm{DE}$ as particular cases, and therefore, have all the advantages of these three algorithms. This is one of the main reasons to explain why we choose the firefly algorithm in this paper.

\section{B. The pseudocode}

Algorithm 1 shows the pseudocode of the original firefly algorithm. The firefly algorithm considers an initial population $\mathcal{P}^{0}$ of $N_{\mathcal{P}}$ individuals $\left\{\mathbf{s}_{i}^{0}\right\}_{i=1, \ldots, N_{\mathcal{P}}}$ initialized randomly. This population size is maintained along the iterations. The firefly search is performed within the while loop (lines 3-10 in Algorithm I), where the following steps are carried out: firstly, the function AlphaNew() modifies the value of parameter $\alpha$ (line 4), although this step is optional in the algorithm, and a constant value can be considered instead. Then, the function EvaluateFFA evaluates the quality of the solution (line 5). All solutions at time $t$ are sorted according to the fitness function (line 6) and the best value is selected and assigned to the global best $\mathbf{s}^{*}$ (line 7). The new positions are computed according 
to the Eqs. (6)-(8), where the fireflies move towards the more attractive individuals of the swarm (line 8). The process is repeated iteratively until the maximum number of iterations is reached. Finally, the best individual at final iteration is selected as the solution of the optimization problem (line 11).

\section{Proposed Methodology}

\section{A. Our Method}

Our method consists of applying the firefly algorithm described in previous section to the border detection problem described in Sect. II. Solving this optimization problem requires to define some important issues. Firstly, we need an adequate representation of the problem. Each individual (firefly), representing a potential solution, corresponds to a vector of the form: $\mathcal{S}_{i}^{g}=\left\{\mathcal{P}_{i}^{g}, \mathcal{W}_{i}^{g}\right\},\left(i=1, \ldots, N_{\mathcal{P}}\right)$ with $\mathcal{P}_{i}^{g}=\left\{\tau_{i, 1}^{g}, \ldots, \tau_{i, \kappa}^{g}\right\} \in[0,1]^{\kappa}$, where the $\left\{\tau_{i, j}^{g}\right\}_{j=1, \ldots, \kappa}$ are strictly increasing parameters, and $\mathcal{W}_{i}^{g}=\left\{\omega_{i, 0}^{g}, \ldots, \omega_{i, \eta}^{g}\right\} \in$ $\left[\omega_{\min }, \omega_{\max }\right]^{\eta+1}$, and the superscript $g$ denotes the generation index. The parametric vectors $\mathcal{P}_{i}^{0}, \mathcal{W}_{i}^{0}$ are initialized with random values; then, the elements in $\mathcal{P}_{i}^{0}$ are sorted in increasing order. Secondly, we consider the fitness function described by the error functional in (2). We remark however, that this fitting error does not take into account the number of data points. To overcome this drawback, we also compute the $R M S E$ (root-mean squared error), given by:

$$
R M S E=\sqrt{\frac{\Upsilon}{\kappa}}
$$

Application of our method according to Algorithm 1 yields new fireflies at each generation, representing the new solutions of our optimization problem. The procedure computes the final values of feature point parameters and weights. Then, inserting them into Eq. (3), we apply least-squares minimization to compute the values of $\left\{\boldsymbol{\Lambda}_{i}\right\}_{i=0, \ldots, \eta}$ according to Eq. (4). The process is performed iteratively for a given number of iterations $\mathcal{T}_{\max }$, until the convergence of the minimization of the error is eventually achieved. The firefly with the best global value for our fitness function is taken as the final solution of our problem.

\section{B. Parameter Tuning}

It is well-known that metaheuristic techniques require proper parameter tuning for good performance [6]. Unfortunately, the choice of suitable parameter values is also strongly problem-dependent. Consequently, our choice is mostly based on a large collection of empirical results. These parameters are:

- the population size, $N_{\mathcal{P}}$ : this value is set up to $N_{\mathcal{P}}=100$ fireflies in all examples of this paper. We also tried larger populations of fireflies but found that our results do not change significantly. Since larger populations mean larger computation times with no remarkable improvement, we found this value to be appropriate in our simulations.

- the number of iterations, $\mathcal{T}_{\max }$. In our simulations, we found that $\mathcal{T}_{\max }=40,000$ is more than enough enough to reach convergence for our examples.
- the initial attractiveness, $\beta_{0}$ : some theoretical results suggest that $\beta_{0}=1$ is a good choice for many optimization problems. We also take this values in this paper, with good results, as it will be discussed in next section.

- the absorption coefficient, $\gamma$ : it is set up to $\gamma=0.5$ in this paper, as this value provides a quick convergence of the algorithm to the optimal solution.

- the potential coefficient, $\mu$ : although any positive value can be used for this parameter, the light intensity varies according to the inverse square law. Therefore, we choose $\mu=2$ accordingly.

- the randomization parameter, $\alpha$. This parameter varies on the interval $[0,1]$, and allow us to determine the degree of randomization introduced in the algorithm. This stochastic component is necessary in order to allow new solutions appear and avoid to getting stuck in a local minimum. However, larger values introduces large perturbations on the evolution of the firefly and, therefore, delay convergence to the global optima. Consequently, it is advisable to select values in between. In this work, we take $\alpha=0.5$.

After the selection of those parameters, the firefly algorithm is executed for the prescribed number of iterations. The firefly with the best (i.e., minimum) fitness value is selected as the best solution to the problem.

\section{Implementation Details}

Regarding the implementation, our computational work has been performed on a personal PC with a $3.4 \mathrm{GHz}$ Intel Core i7 processor and $16 \mathrm{~GB}$ of RAM. The source code has been implemented by the authors in the programming language of the popular numerical program Matlab, version $2018 \mathrm{~b}$.

\section{EXPERIMENTAL RESUlts}

\section{A. Benchmark and Results}

Our method has been applied to several examples of skin lesion images obtained from a digital image repository publicly available for research purposes. In this paper we analyze only two of them (labelled as Example I and Example II) because of limitations of space. They correspond to two medical images of melanomas, displayed in the (top-left) pictures of Figs. 1 and 2, respectively. From the images, a collection of 75 and 127 feature points respectively have been selected by a specialist and joined with a polyline. The medical images along with the feature points connected with the polylines are displayed in the (top-right) pictures of Figs. 1 and 2. We applied our method to these examples by using rational Bézier curves of different degrees, and selecting those minimizing the least-squares functional in Eq. (2). The best fitting rational Bézier curves obtained with our method are displayed as blue solid lines in the (bottom-left) pictures of Figs. 1 and 2, respectively. The pictures also display the original and the reconstructed feature points as empty red circles and blue stars, respectively. Finally, the convergence diagram of the RMSE given by Eq. (9) over the generations is shown in the (bottomright) pictures of both figures. 

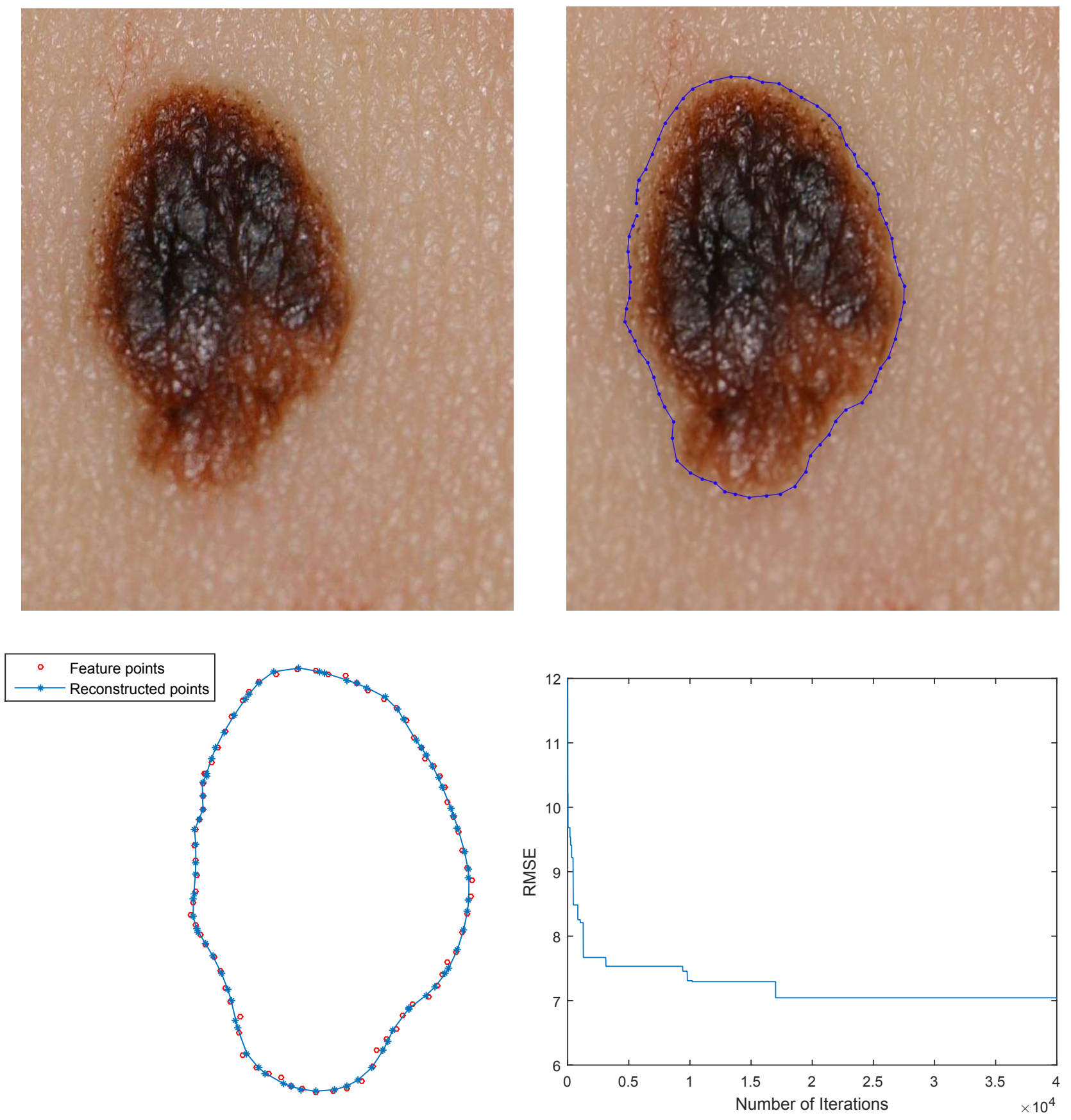

Fig. 1. Example I: (top-left) original melanoma image; (top-right) melanoma image and polyline connecting the feature points; (bottom-left) original and reconstructed feature points; (bottom-right) convergence diagram.

From the pictures we can see that the method yields a good fitting of the feature points for both examples. This fact is particularly evident in the bottom-left pictures, where the good matching between the original and the reconstructed feature points for both examples is clearly visible. Our visual observations of this good fitting are confirmed by our numerical results, where we obtain an error value of 7.0324 for the first example and 16.2938 for the second one. We also noticed that the approximation is not optimal yet, as expected from an approximation method. In particular, the original data are visually more oscillating than the reconstructed curve in both cases. We remark, however, that a perfect matching (i.e. interpolation) between the original and the reconstructed features points is not actually required for clinical diagnosis at this stage.

\section{B. Comparative Analysis}

It is always convenient to perform a comparative analysis of our method with other approaches described in the literature. 

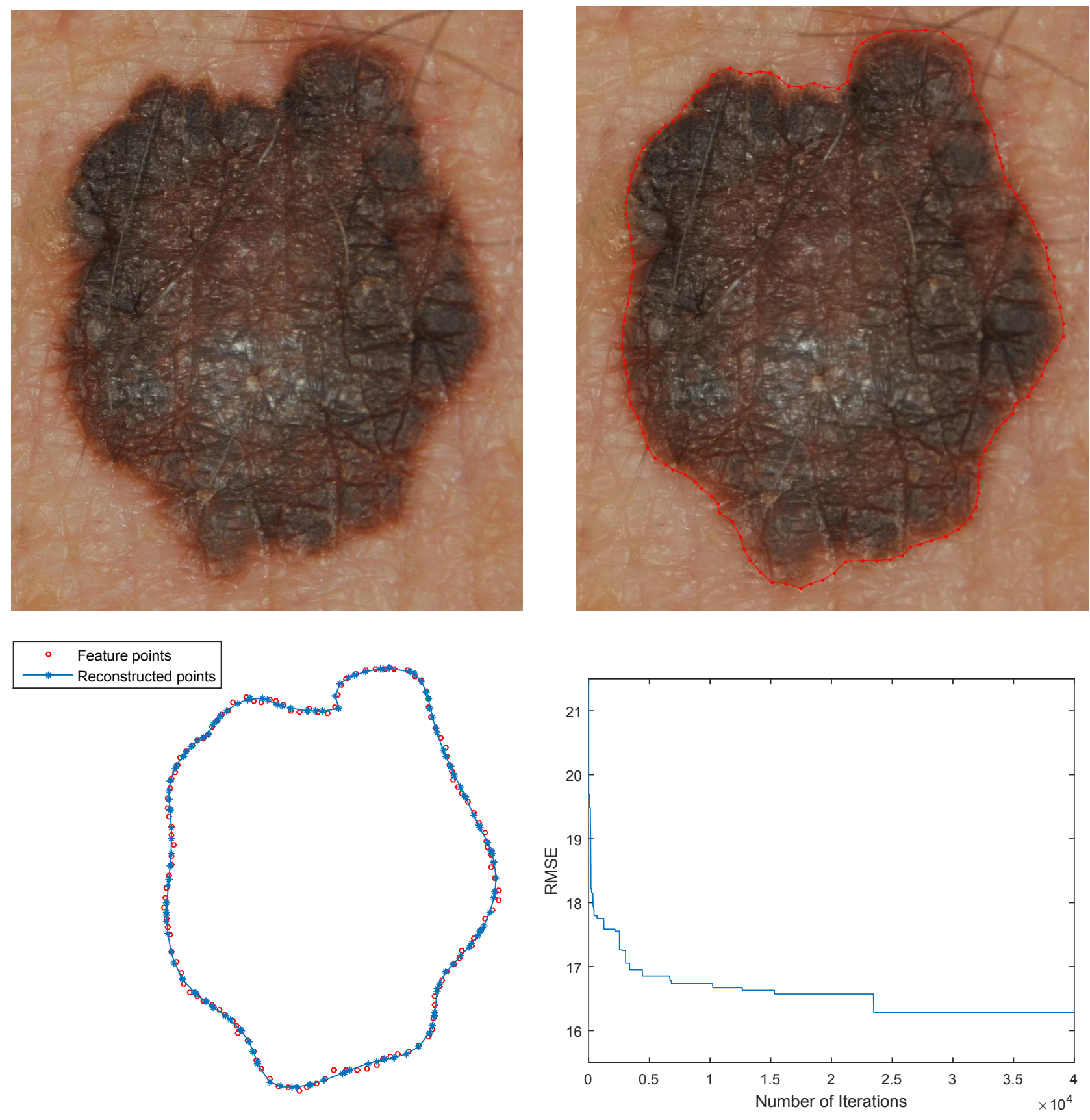

Fig. 2. Example II: (top-left) original melanoma image; (top-right) melanoma image and polyline connecting the feature points; (bottom-left) original and reconstructed feature points; (bottom-right) convergence diagram..

Unfortunately, no previous references addressed this problem with rational curves, so our analysis is based on the comparison with the polynomial case with the firefly algorithm. Table I shows the values of the RMSE obtained with the polynomial approach and our rational approach for the two examples in this paper. For fair comparison, both approaches are addressed with the firefly algorithm for the same parameter configuration, including the number of iterations and the degree of the curve. As shown in the table, the rational approach outperforms the polynomial one for the two examples in this paper. Although the benchmark used here is too small to draw firm conclusions on a general basis, these results have also been validated by other examples from the same digital repository not discussed here because of limitations of space.

\section{CONCLUSions \& FUture Work}

In this paper, we address the problem of obtaining the border curve of melanomas and other skin lesions from medical images through rational Bézier curves. This is an important task for image segmentation with relevant applications in medical 
TABLE I

COMPARATIVE RESULTS OF THE RMSE WITH THE POLYNOMIAL APPROACH AND THE RATIONAL APPROACH (OUR METHOD) FOR THE TWO EXAMPLES IN THIS PAPER.

\begin{tabular}{|l|c|c|}
\hline \hline & Example I & Example II \\
\hline Polynomial approach: & 10.6212 & 19.5744 \\
\hline Rational approach: & 7.0324 & 16.2938 \\
\hline
\end{tabular}

diagnosis of skin diseases. To the best of our knowledge, this is the first work in the field considering rational functions instead of the classical (and much simpler) polynomial functions. Our method is based on the firefly algorithm, a popular bio-inspired swarm intelligence approach for optimization. Experimental results on two examples of medical images of melanomas obtained from a public medical imaging repository show that the proposed method performs well, yielding approximating shapes with an acceptable accuracy from the practical applications. Our results also show that this rational approach outperforms the polynomial approach and can be applied to medical images without further pre/post-processing.

In spite of these good results, the method has also some limitations. For instance, the method requires many iterations to converge, leading to large $\mathrm{CPU}$ times. From the convergence diagrams, we can see that the method is stuck in local minima for long periods of time without any improvement. We think the coupling of this method with a local search procedure could be helpful to avoid stagnation, thus reducing the computation times significantly. In addition, we are interested to expand our benchmark for a more detailed analysis of our method. Our future work also includes carrying out a larger and deeper comparative work involving other evolutionary computation methods in the pool of methods for comparison. Application of this technique for other problems in medical imaging is also part of our plans for future work in the field.

\section{ACKNOWLEDGMENT}

The authors thank the financial support from the project PDE-GIR of the European Union's Horizon 2020 research and innovation programme under the Marie Sklodowska-Curie grant agreement No 778035. The first two authors also thank financial support from the Spanish Ministry of Science, Innovation and Universities (Computer Science National Program) under grant \#TIN2017-89275-R of the Agencia Estatal de Investigación and European Funds EFRD (AEI/FEDER, UE).

\section{REFERENCES}

[1] Q. Abbas, M. E. Celebi, I. F. Garcia, M. Rashid: Lesion border detection in dermoscopy images using dynamic programming, Skin Research and Technology, 17(1), 91-100 (2011).

[2] Abbas, A.A., Guo, X., Tan, W.H., Jalab, H.A.: Combined spline and B-spline for an improved automatic skin lesion segmentation in dermoscopic images using optimal color channel. Journal of Medical Systems, 38, 80-80 (2014).

[3] Argenziano, G., Soyer, H.P., De Giorgi, V.: Dermoscopy: A Tutorial. EDRA Medical Publishing \& New Media (2002).

[4] M. E. Celebi, H. Iyatomi, H., Schaefer, G., Stoecker, W.V.: Lesion border detection in dermoscopy images. Computerized Medical Imaging and Graphics, 33(2) 148-153 (2009).
[5] Dierckx, P.: Curve and Surface Fitting with Splines. Oxford University Press, Oxford (1993)

[6] Engelbrecht, A.P.: Fundamentals of Computational Swarm Intelligence. John Wiley and Sons, Chichester, England (2005).

[7] Farin, G.: Curves and surfaces for CAGD (5th ed.). Morgan Kaufmann, San Francisco (2002).

[8] Friedman, R.J., Rigel, D.S., Kopf, A.W.: Early detection of malignant melanoma: The role of physician examination and self-examination of the skin. Cancer Journal for Clinicians, 35(3), 130-151 (1985).

[9] Gálvez, A., Iglesias A., Puig-Pey J.: Iterative two-step genetic-algorithm method for efficient polynomial B-spline surface reconstruction. Information Sciences, 182(1) 56-76 (2012).

[10] Gálvez A., Iglesias A.: Particle swarm optimization for non-uniform rational B-spline surface reconstruction from clouds of 3D data points. Information Sciences, 192(1), 174-192 (2012).

[11] Gálvez A., Iglesias A.: A new iterative mutually-coupled hybrid GAPSO approach for curve fitting in manufacturing. Applied Soft Computing, 13(3), 1491-1504 (2013).

[12] Gálvez, A., Iglesias, A.: Firefly algorithm for explicit B-spline curve fitting to data points. Mathematical Problems in Engineering, 2013, Article ID 528215, 12 pages (2013).

[13] Gálvez, A., Iglesias, A.: New memetic self-adaptive firefly algorithm for continuous optimisation. International Journal of Bio-Inspired Computation, 8(5), 300-317 (2016).

[14] Gálvez, A., Fister, I., Fister Jr., I., Osaba, E., Del Ser, J., Iglesias, A.: Automatic fitting of feature points for border detection of skin lesions in medical images with bat algorithm. Studies in Computational Intelligence, 798, 357-368 (2018).

[15] Gálvez, A., Iglesias, A.: Computational intelligence CSA-based approach for machine-driven calculation of outline curves of cutaneous melanoma. Proc. of Int. Conf. on Cyberworlds, CW-2018, Singapore. IEEE CS Press, 270-275 (2018).

[16] Garnavi, R. Aldeen, M., Celebi, M.E., Varigos, G., Finch, S.: Border detection in dermoscopy images using hybrid thresholding on optimized color channels. Computerized Medical Imaging and Graphics, 35(2), 105-115 (2011).

[17] Iglesias, A., Gálvez, A., Collantes, M.: Multilayer embedded bat algorithm for B-spline curve reconstruction. Integrated Computer-Aided Engineering, 24(4), 385-399 (2017).

[18] Iglesias, A., Gálvez, A., Avila, M.: Immunological approach for full NURBS reconstruction of outline curves from noisy data points in medical imaging. IEEE/ACM Transactions on Computational Biology and Bioinformatics, 15(8), 1929-1942 (2018).

[19] Korotkov, K., Garcia, R.: Computerized analysis of pigmented skin lesions: a review. Artificial Intelligence in Medicine, 56, 69-90 (2012).

[20] Ma, Z., Tavares, J.M.: A novel approach to segment skin lesions in dermoscopic images based on a deformable model. IEEE Journal of Biomedical and Health Informatics, 20, 615-623 (2016).

[21] Machado, D.A., Giraldi, G., Novotny, A.A.: Multi-object segmentation approach based on topological derivative and level set method. Integrated Computer-Aided Engineering, 18, 301-311 (2011).

[22] Nachbar, F., Stolz, W., Merkle, T., Cognetta, A.B., Vogt, T., Landthaler, M., Bilek, P., Braun-Falco, O., Plewig, G.: The ABCD rule of dermatoscopy. High prospective value in the diagnosis of doubtful melanocytic skin lesions. Journal American Academy of Dermatology, 30(4), 551-559 (1994).

[23] Pathan, S., Prabhu, K.G., Siddalingaswamy, P.C.: Techniques and algorithms for computer aided diagnosis of pigmented skin lesions - a review. Biomedical Signal Processing and Control, 39, 237-262 (2018).

[24] Piegl, L., Tiller, W.: The NURBS Book, Springer Verlag, Berlin Heidelberg (1997).

[25] Schmid, P.: Segmentation of digitized dermatoscopic images by twodimensional color clustering. IEEE Transactions on Medical Imaging, 18(2), 164-171 (1999).

[26] Yang, X.S.: Firefly algorithms for multimodal optimization. Lectures Notes in Computer Science, 5792,169-178 (2009).

[27] Yang, X.S.: Firefly algorithm, stochastic test functions and design optimisation. Int. J. of Bio-Inspired Computation, 2(2) 78-84 (2010).

[28] Yang, X.-S.: Nature-Inspired Metaheuristic Algorithms (2nd. Edition). Luniver Press, Frome, UK (2010).

[29] Zhou, H., Schaefer, G., Sadka, A., Celebi, M.E.: Anisotropic mean shift based fuzzy c-means segmentation of dermoscopy images. IEEE Journal of Selected Topics in Signal Processing, 3(1) 26-34 (2009). 